Strom in Flußrichtung durch die unbelichtete Sperrschicht einen solchen Photostrom zu, daß der Defektelektronen-Strom an der Grenze Metall/ Halbleiter in beiden Fällen gleich ist, so werden sich die betreffenden Spannungen annähernd entsprechen. Daher besteht zwischen der Photokenn-

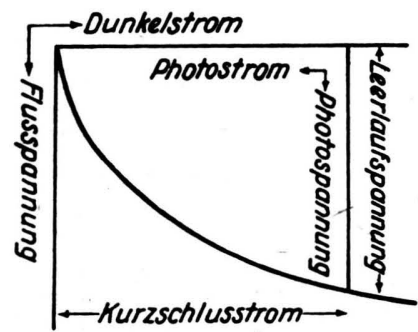

Abb. 4. Näherungsweiser Zusammenhảng zwischen der Kennlinie der unbelichteten Sperrschicht und der Photokennlinie.

linie und der Dunkelkennlinie in Flußrichtung der in Abb. 4 dargelegte näherungsweise Zusammenhang. Ein entsprechender Zusammenhang ist für den Fall der Leerlaufspannung bereits von $\mathrm{Mott}^{5}$ angegeben worden.
6. Wir haben bereits erwähnt, daß für die Ausbildung einer nennenswerten Sperrschicht Störstellen-Halbleitung, und zwar entweder praktisch ausschließliche Überschußhalbleitung oder praktisch ausschließliche Defekthalbleitung, notwendig ist. Für das Auftreten des Sperrschicht-Photoeffekts kommt die Forderung hinzu, daß durch Lichtabsorption Elektronen und Defektelektronen ausgelöst werden. Würden nämlich durch Lichtabsorption ortsfeste Zentren (z. B. Verunreinigungen) ionisiert, so müßten sich im stationären $\mathrm{Zu}$ stand lichtelektrische Dissoziation und Rekombination an jeder Stelle (auch in der Sperrschicht!) entsprechen, und es könnten sich keine ortsabhängigen Ströme ausbilden. Diese sind aber gerade für den Sperrschicht-Photoeffekt wesentlich! Die Einstellung des stationären Zustands bei Belichtung erfolgt in so einem Fall in der Weise, daß durch Lichtabsorption zunächst so viele Zentren ionisiert werden, und die Zahl der nicht ionisierten Zentren so lange abnimmt, bis die dadurch verminderte $\mathrm{Ab}$ sorption der geringen Rekombination in der Sperrschicht entspricht.

\title{
Über den Zerfall übersättigter Magnesium-Mischkristalle
}

\author{
Von Walter Bulian und Eberhard Fahrenhorst
}

Aus dem Metall-Laboratorium der Wintershall A.-G. in Heringen/Werra

(Z. Naturforschg. 1, 263-267 [1946]; eingegangen am 8. April 1946)

\begin{abstract}
Die beim Zerfall von Magnesium-Aluminium- Mischkristallen entstehenden Entmischungsgefüge Eutektoid und gerichtete Ausscheidungen besitzen ihrer Entstehungstemperatur nach keine scharfe Temperaturgrenze. Mg-Mischkristalle mit 8 anderen Legierungskomponenten zerfallen beim Anlassen unter Bildung gerichteter Ausscheidungen, die bei niedrigen Anlaßtemperaturen allein auf der Basis, bei höheren auch auf anderen möglichen Ausscheidungsebenen des Mg-Kristalls liegen. Eutektoid wurde niemals bei ihnen beobachtet. Die ,gerichteten Ausscheidungen“ und das „Eutektoid“ entsprechen den mikroskopisch homogenen und mikroskopisch inhomogenen Ausscheidungen.
\end{abstract}

$\ddot{U}$ bersättigte Magnesium-Aluminium-Mischkristalle mit mehr als 4\% Al zerfallen beim Erwärmen auf etwa $200^{\circ}$ oder auch beim langsamen Abkühlen erstarrter Schmelzen in das schon mehrfach beschriebene eutektoidähnliche Gefüge ${ }^{1,2}$, für das wir in ${ }^{1}$ die Bezeichnung, Eutektoid“ benutzten ${ }^{3}$. H. Vosskühle ${ }^{4}$ weist mit Recht darauf hin, daß

1 W. Bulian u. E. Fahrenhorst, Metallographie des Magnesiums, Springer-Verlag, Berlin 1942, S. $19 \mathrm{ff}$.

2 W. Bulian u. E. Fahrenhorst, Z. Metallkunde 34, 285 [1942]. diese Bezeichnung denn doch echtem Eutektoid vorbehalten bleiben soll, und schlägt statt dessen die Bezeichnung: Entmischungsgefüge, Anlaßgefüge oder sekundäre Ausscheidungen vor. Von diesen scheint uns nun der erste Ausdruck der beste zu sein, wenn man nicht die Ausscheidungslamellen allein, sondern diese zusammen mit den

3 Über den Unterschied zwischen der besprochenen Ausscheidungsform und echtem Eutektoid siehe besonders unter ${ }^{2}$.

4 Aluminium 24, 407 [1942]. 
zwischen ihnen liegenden $\alpha$-Mischkristallen, also die gesamte perlitartige Struktur, als welche sie auch schon ${ }^{5}$ bezeichnet worden ist, benennen will. Nun gilt er aber auch für die bei höheren Anlaßtemperaturen entstehenden Gefügearten (Abb.2) mit gerichteten Ausscheidungen. Um also die Unterscheidung dieser Gefügebilder beizubehalten, die neuerdings bestätigt wurde ${ }^{\mathbf{b}}$, der Ausdruck „Perlit" aber wohl doch dem entsprechenden Gefüge des Eisen-Kohlenstoff-Systems vorbehalten bleiben soll, wollen wir im folgenden trotz der oben geäußerten Bedenken bei dem Ausdruck „Eutektoid“ für das ungerichtete, lamellare, perlitartige Entmischungsgefüge des Systems Magnesium-Aluminium bleiben.

Auf die Bezeichnungsschwierigkeiten bei charakteristischen Gefügearten bei NE-Legierungen ist schon einmal hingewiesen worden ${ }^{7}$. Es war der Vorschlag gemacht worden, für derartige Gefügeausbildungen, wie sie besonders (wie im vorliegenden Fall) bei Wärmebehandlung auftreten, Forschernamen analog dem Vorgang beim Eisen zu verwenden. Auch wir halten diesen Weg für den besten, möchten aber dahingehende Einzelvorschläge berufenerem Munde überlassen.

\section{Weitere Versuche im System Magnesium-Aluminium}

Wie schon gezeigt worden war $^{2}$, erfolgen bei höheren Anlaßtemperaturen die Ausscheidungen aus den übersättigten Magnesium-AluminiumMischkristallen in gerichteter Anordnung. Es war nun noch zu untersuchen, ob zwischen dieser und der eutektoiden Ausscheidungsform eine eindeutige Temperaturgrenze besteht.

Die Versuche mit etwa 7-proz. Mg-Al-Legierungen, die etwa 8 Tage bei $420^{\circ}$ homogenisiert worden waren und damit nach ${ }^{8}$ völlig homogen waren, erbrachten den Nachweis, daß das Eutektoid oberhalb $280^{\circ}$ nicht mehr auftritt. Die bei noch höheren Temperaturen allein vorkommenden gerichteten Ausscheidungen treten hingegen neben dem Eutektoid bis herab zu $140^{\circ}$ auf, und zwar derart, daß zwischen $140^{\circ}$ und $300^{\circ}$ vorzugsweise - und im unteren Temperaturbereich allein - parallele Ausscheidungen auf der Basis des Mg-Kristalls auf-

5 K. R. M e is sne r, J. Inst. Metals 38, 195 [1927].

6 M. A. F o x, Ind. 1944, 114.

7 Metal. Ind. [London] 1942, 202.

8 W. Bulian u. E. Fahrenhorst, Z. Metallkunde 36, 20 [1944]. treten, während oberhalb $300^{\circ}$ auch andere Ausscheidungsebenen, vorzugsweise Prismen und Pyramidenflächen, benutzt werden, wie früher ${ }^{2}$ genauer ausgeführt worden ist.

Die von M. A. Fox ${ }^{6}$ mit etwa $200^{\circ}$ als obere Grenze für die Entstehung des Eutektoids angegebene Temperatur ist also noch erheblich zu niedrig gegriffen.

Das Bild 1 zeigt das Entmischungsgefüge einer 6,5-proz. Mg-Al-Legierung, die 50 Stdn. bei $140^{\circ}$ angelassen worden war. Die abgebildete Stelle des Schliffes zeigt rein parallele Ausscheidungen mit Ausnahme einer kleinen Stelle in der Mitte, an der sich von den Korngrenzen ausgehend etwas Eutek-

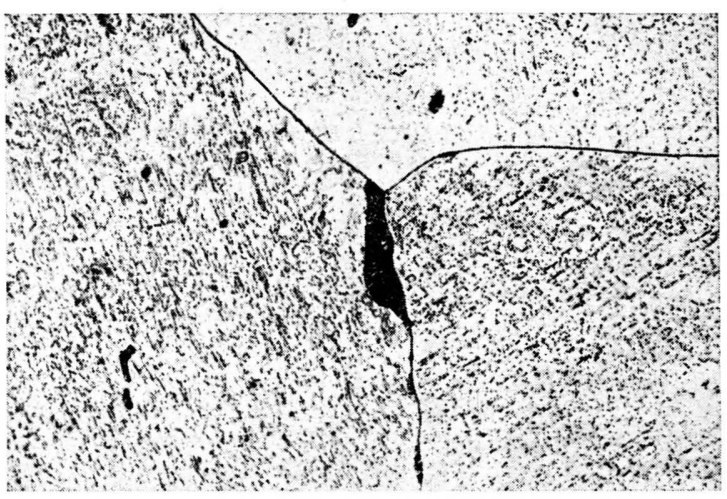

Abb. 1. $\mathrm{Mg}+6,5 \% \mathrm{Al}, 50 \mathrm{Stdn}$. bei $140^{\circ}$ angelassen, in der Mitte Eutektoid, sonst gerichtete Ausscheidungen. Vergr. 300/1.

toid bildete. Im übrigen war das Versuchsstück an anderen Stellen auch mit größeren Eutektoidgebieten durchsetzt; diese beiden Ausscheidungsformen kommen also bei dieser Temperatur zusammen vor. Demnach existiert zwischen den beiden Entmischungsgefügen keine scharfe zweiseitige Temperaturgrenze.

Es entstehen also bei der Entmischung von übersättigten Mg-Al-Mischkristallen die Gefügearten: Eutektoid, gerichtete Ausscheidungen in paralleler Streifenanordnung und stäbchenförmige Ausscheidungen, die unter Winkeln von 60 und $90^{\circ} \mathrm{zu}-$ einander liegen (1. c. ${ }^{2}$, Abb. 6 u. 10), und zwar entstehen die beiden ersten bei tiefen, die letztere allein bei höheren Anlaßtemperaturen.

Ausgehend von einem weiter unten ausführlicher besprochenen Hinweis von U. Dehling er wurde noch untersucht, wieweit die beiden Hauptgruppen der Ausscheidungsformen (Eutektoid und gerichtete Ausscheidungen) vom Grad der Übersättigung 
des Mischkristalls abhängen. Es zeigte sich, daß bei geringer Übersättigung $(4 \% \mathrm{Al})$ ausschließlich gerichtete Ausscheidungen auftraten, während bei größerer $\mathrm{Al}-\mathrm{Konzentration}(6 \% \mathrm{Al})$ bei $220^{\circ}$ neben diesen auch Eutektoid auftrat. Zur Eutektoidentstehung gehört also eine größere Übersättigung. Das steht in Übereinstimmung mit der Tatsache, daß es bei nicht homogenisiertem Material, bei dem also bei selbst sehr niedrigem Gesamt-AlGehalt $(>3,5 \%)$ örtlich hohe Konzentrationen vorkommen können, so leicht auftritt.

Bekanntlich ist die Geschwindigkeit der Entmischung stark vergrößert, wenn ihr eine plastische Verformung vorangeht. Das äußert sich (l. c. ${ }^{1}$, S. 81, Abb. 71) bei Magnesium-Aluminium-Legierungen darin, daß hierbei entstandene $Z$ willinge äußerst leicht Ausscheidungen zeigen. Es gelang uns nun nicht, Richtungen, auf denen die pünktchenförmigen Ausscheidungen von $\mathrm{Al}_{2} \mathrm{Mg}_{3}$ innerhalb der Zwillinge angeordnet sein könnten, mikroskopisch festzustellen. Sicher gehört diese Ausscheidungsform aber nicht zu der Art des Eutektoids, zumal sie im ganzen Zwillingskristall auf einmal sichtbar wird und ein Wachsen der Entmischungszonen, wie es für das Eutektoid typisch ist, nicht beobachtet wurde.

Die Ausscheidungsformen bei Mg-Mischkristallen mit anderen Legierungskomponenten

Es wurden nun weiter die Entmischungsvorgänge an Magnesium-Mischkristallen mit einer Reihe von Metallen als Legierungskomponenten untersucht, die alle eine mehr oder weniger starke Abhängigkeit ihrer Löslichkeit im Magnesium von der Temperatur aufwiesen. Es waren dies, nach fallender Löslichkeit (in AT\% geordnet) Thallium, Blei, Zink, Silber, Zinn, Calcium, Wismut und Mangan. Dabei war von besonderem Interesse die Frage, ob die Mischkristalle des Magnesiums mit diesen Komponenten beim Zerfall ebenfalls wie bei Mg-Al zwei Arten von Ausscheidungen, Eutektoid und gerichtete Ausscheidungen, bilden. Es wurde so vorgegangen, daß Legierungen mit verhältnismäßig hoher Mischkristall-Konzentration zur leichteren Entmischbarkeit im Gußzustand nur kurz homogenisiert und dann langsam im Ofen gekühlt wurden. Außerdem wurden auch stark warmverformte Proben in gleicher Weise behandelt und schließlich wurden alle Proben noch einer lang- zeitigen Homogenisierungsglühung dicht unter der Temperatur der jeweiligen Eutektikalen sowie anschließend einer Wärmebehandlung bei $180^{\circ}, 200^{\circ}$ und $300^{\circ}$ unterzogen. Als Ergebnis sei vorweggenommen, daß diese unterschiedlichen Versuchsbedingungen im Gegensatz zu den Verhältnissen im System Mg-Al keine Unterschiede im Aussehen

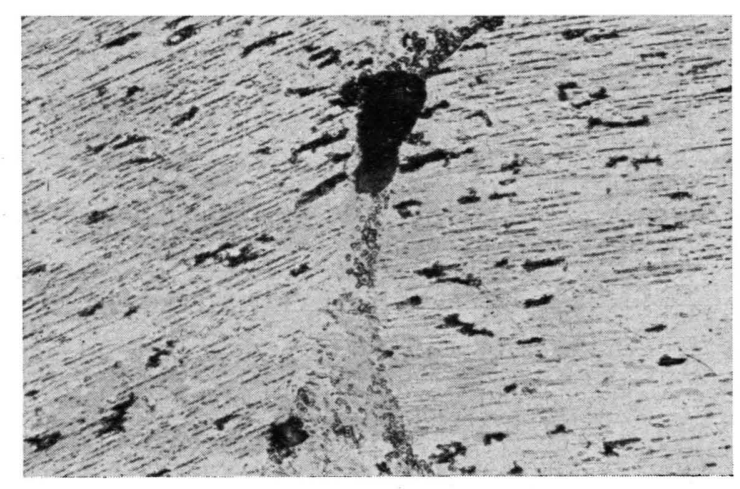

Abb. 2. Mg + 60\% Tl, 2 Tage bei $200^{\circ}$ angelassen. Vergr. 250/1.

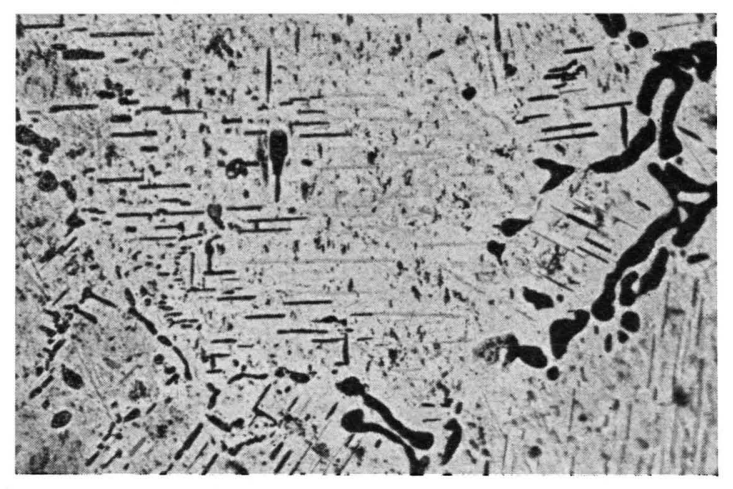

Abb. 3. $\mathrm{Mg}+40 \% \mathrm{~Pb}, 24 \mathrm{Stdn}$. bei $300^{\circ}$ angelassen. Vergr. 350/1.

der Entmischungsgefüge erbrachte. Die Abb. $2-5$ bringen das Ergebnis der Untersuchung.

Magnesium-Mischkristalle mit Thallium zeigen bei einer Anlaßbehandlung das in Abb. 2 wiedergegebene Gefüge. Man erhält parallel liegende Ausscheidungen von $\mathrm{Mg}_{3} \mathrm{Tl}_{2}$, Eutektoid wurde nie beobachtet. Bei höherer Anlaßtemperatur treten außerdem andere Ausscheidungsrichtungen hinzu, die unter Winkeln von 60 und $90^{\circ}$ zueinander stehen. Nicht in Übereinstimmung hiermit steht die Behauptung von W.Hume-Rothery und G.V. Raynor ${ }^{\theta}$, wonach die Magnesium-Thallium-

a J. Inst. Metals 63, 225 [1938]. 
Legierungen beim Zerfall homogener Legierungen keine „Widmannstätten-Struktur“ zeigen.

Abb. 3 gibt das zerfallene Gefüge von Magnesium-Mischkristallen mit Blei wieder. Die Ausscheidungen von $\mathrm{Mg}_{2} \mathrm{~Pb}$ liegen bei dem bei niederen Temperaturen angelassenen Stück nur parallel zueinander, während bei höheren Anlaßtemperaturen auch wie schon früher bei Mg-Al (a.a.O.) Aus-

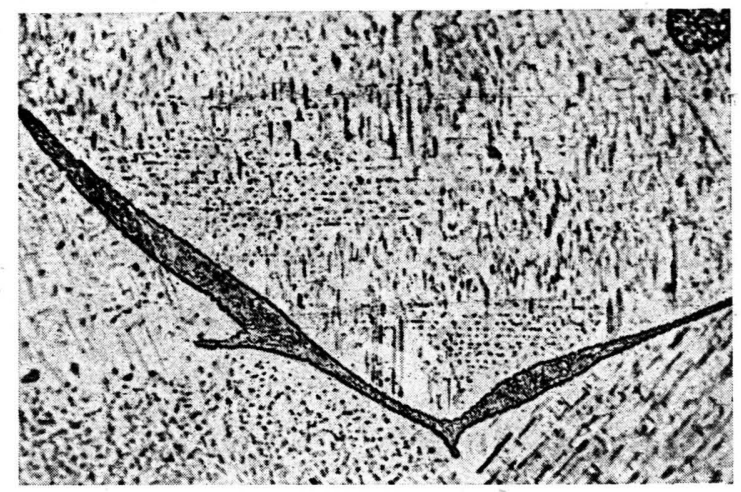

Abb. $4 . \mathrm{Mg}+12 \% \mathrm{Zn}$, bei $300^{\circ}$ angelassen. Vergr. 600/1.

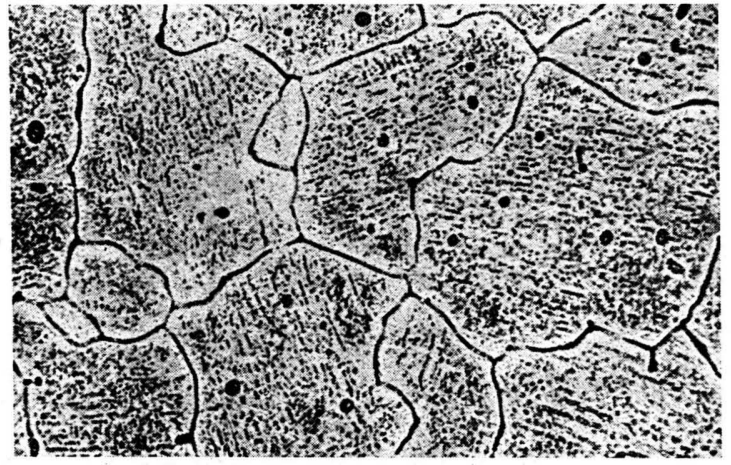

Abb. 5. $\mathrm{Mg}+0,9 \% \mathrm{Ca}$, bei $200^{\circ}$ angelassen. Vergr. $175 / 1$.

scheidungsrichtungen beobachtet werden können, die einen Winkel von $90^{\circ}$ zueinander bilden.

Zink-Ausscheidungen sind verhältnismäßig schwierig zu erhalten. Das Zink geht in Magnesium sehr leicht in feste Lösung und scheidet sich bei den in technischen Legierungen vorhandenen Mengen bis zu 4\% überhaupt nicht aus. Abb. 4 zeigt Ausscheidungen von $\mathrm{MgZn}$, die großenteils parallel zueinander, zum Teil aber auch senkrecht zur Hauptausscheidungsrichtung liegen.

Auch Silber zeigt nur gerichtete Ausscheidungen von $\mathrm{Mg}_{3} \mathrm{Ag}$, Eutektoid wurde auch bei ihm unter keinen Versuchsbedingungen gefunden. Ebenfalls rein parallel zueinander liegen die Ausscheidungen von $\mathrm{Mg}_{2} \mathrm{Sn}$ aus Magnesium-Zinn-Mischkristallen. Abb. 5 gibt eine Legierung mit knapp 1\% Calcium wieder. Die Ausscheidungen aus den Mischkristallen erfolgen auch hier wieder alle gerichtet, und zwar wie immer bei Vorliegen nur einer Richtung auf der Basisebene des Magnesiumkristalls. Die Wärmebehandlung bestand in einem 7-tägigen Anlassen bei $180^{\circ}$, Eutektoid wurde niemals beobachtet.

Auch das Wismut bietet nichts Neues, die Ausscheidungen von $\mathrm{Mg}_{3} \mathrm{Bi}_{2}$ liegen wie immer bei tieferen Anlaßtemperaturen nur parallel zueinander, bei höheren treten außerdem unter $60^{\circ}$ zueinander liegende Ausscheidungsrichtungen auf.

Es war schon früher (l. c. ${ }^{1}$, S. 41) darauf hingewiesen worden, daß Mangan sich immer auf der Basis des Magnesiumkristalls abscheidet. Bei sehr hohen Mangankonzentrationen $(>4 \%)$ werden ebenfalls zusätzlich neue Ausscheidungsrichtungen beobachtet, die wieder wie im vorher besprochenen Fall unter einem Winkel von $60^{\circ}$ zueinander liegen.

\section{Diskussion der Ergebnisse}

Während also, wie im Abschnitt $\mathrm{Mg}$-Al gezeigt wurde, das Aluminium sich aus dem Magnesium in 3 verschiedenen Entmischungsgefügen ausscheidet, besitzen die im vergangenen Abschnitt behandelten Legierungskomponenten bei der Ausscheidung aus dem Magnesium-Mischkristall nur 2 Arten von Ausscheidungsformen; in keinem einzigen Fall wurde diebeim Aluminium so stark vorherrschende Form des Eutektoids mit ungerichteten lamellaren Ausscheidungen gefunden.

Man kann beim Zerfall übersättigter Magnesiumkristalle demnach folgende Ausscheidungsarten unterscheiden:

1. a) gerichtete Ausscheidungen als parallele Basisstreife,

b) gerichtete Ausscheidúngen mit zusätzlichen Ausscheidungsebenen auf Prismen- und Pyramidenflächen;

\section{Eutektoid.}

Hierbei verläuft die zweite Art, wie früher gezeigt wurde ${ }^{1,2}$ so, daß sie an den Korngrenzen beginnend beim weiteren Zerfall fortschreitet, während die erste Art, worunter $1 \mathrm{a}$ ) und $1 \mathrm{~b}$ ) verstanden sein soll, gleichzeitig über ganze Kornflächen pünktchenförmig auftretend, sozusagen auf ein- 


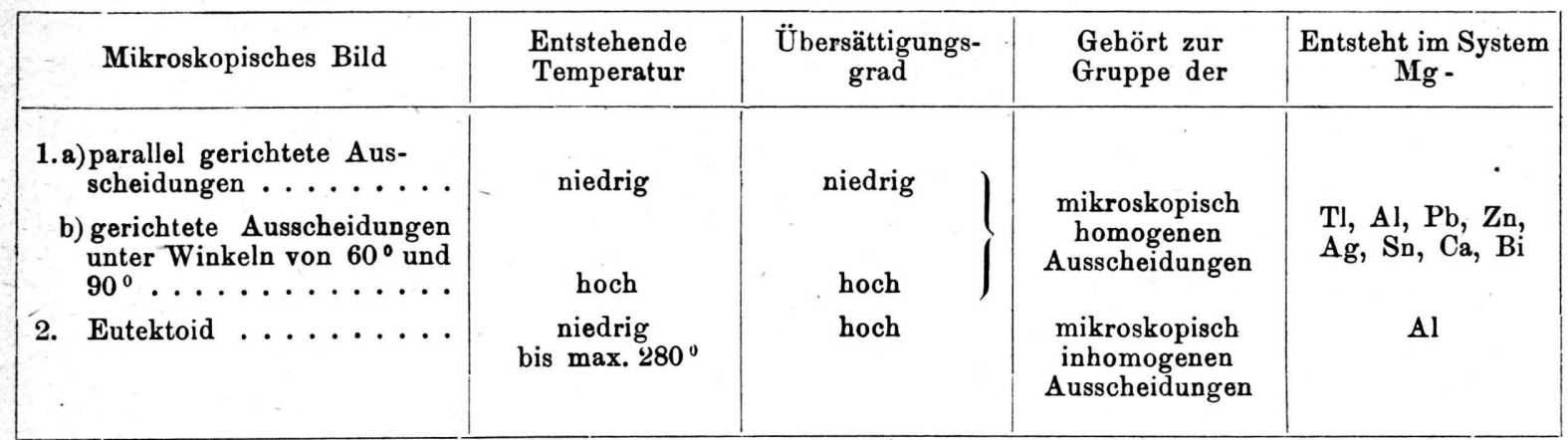

Tab. 1. Überblick über die Versuchsergebnisse.

mal erscheint und im weiteren Verlauf des Zerfallvorganges sich nur noch durch Zusammenballung vergröbert. Diese beiden Ausscheidungsarten sind von W. Gerlach ${ }^{10}$ und U. Dehlinger ${ }^{11}$ als mikroskopisch homogene und mikroskopisch inhomogene Ausscheidung bezeichnet worden. Dabei ist für die mikroskopisch inhomogene Ausscheidung - in unserem Fall das Eutektoid - das Wachstum des zerfallenden Gebietes, also die Lokalisierung des Ausscheidungsvorganges auf das Randgebiet charakteristisch, was U. Dehlinger mit einer autokatalytischen Beschleunigung erklärt, während nach dem gleichen Autor die erste Art sich durch die Bildung eines zunächst hochdispersen Gemenges, welches über den ganzen Kristall hinweg gleichmäßig vorliegt, erklärt.

Wie im Falle des Mg-Al gezeigt wurde, lassen sich die Ausscheidungen innerhalb von stark verformten Bereichen, wie $\mathrm{Z}_{\text {willingskristalle, nicht in die }}$ Gruppen ,gerichtete Ausscheidungen“ und „Eutektoid" einreihen. Trotzdem muß auch diese Ausscheidungsart zur Gruppe der mikroskopisch homogenen Ausscheidungen gerechnet werden, da für sie das Charakteristikum des Eutektoids, das Wachsen, nicht zutrifft, vielmehr ebenso wie bei mikroskopisch homogenen Ausscheidungen das gesamte Gebiet, sowie eine Entmischung mikroskopisch merkbar wird, von Ausscheidungen durchsetzt wird. Die Ausscheidungen in den Zwillingen sind also mikroskopisch homogene Ausscheidun-

10 W. Gerlach, Z. Metallkunde 29, 102, 124, 145 [1937].

11 U. Dehlinger, Chemische Physik d. Metalle u. Legierungen, Leipzig 1939, S. $111 \mathrm{ff}$.

${ }_{12}$ W. Hu me-R othery u. G. V. R a y nor, J. Inst. Metals 63, 219 [1938], fanden bei Mg-In ebenfalls nur gerichtete Ausscheidungen. gen, die nach U. De hling e ${ }^{10}$ und W. Gerla $\operatorname{ch}^{11}$ uneinheitlicher geworden sind, aber noch immer nicht mit mikroskopisch inhomogenen Ausscheidungen zu verwechseln sind.

Nach den vorliegenden Ergebnissen zeigt sich also die zweite Art der Ausscheidungsformen in der oben gegebenen Zusammenstellung nur beim Zerfall übersättigter Magnesium-AluminiumMischkristalle, während die erste Art bei Mg-Al und allen anderen untersuchten Legierungspartnern vorkommt ${ }^{12}$. Das Eutektoid ist demnach als Zerfallsgefüge ein auf das $\mathrm{Al}$ beschränkter einmaliger Sonderfall ${ }^{13}$. Tab. 1 gibt einen Überblick über die Versuchsergebnisse in Verbindung mit der Einteilung von U. Dehlinger.

Eine Erklärung für diese Einmaligkeit kann aus dem vorliegenden Versuchsmaterial noch nicht gefunden werden. Insbesondere hängt die Eutektoidentstehung nicht, wie nach den Untersuchungen über den Einfluß des Übersättigungsgrades zu vermuten wäre, von der Höhe der Übersättigung, d. h. also vom Verlauf der Löslichkeitslinie ab. Die Löslichkeitslinie des Al im Mg liegt, in Atom-\% aufgetragen, zwischen denen des noch stärker löslichen Thalliums und des Bleis, die beide, zusammen mit den noch beträchtlich löslichen $\mathrm{Zn}$ und Ag, bei ähnlich starker Abhängigkeit der Löslichkeit von der Temperatur erhebliche Übersättigungen bei sowohl mehr als auch weniger gelöster $\mathrm{Ge}-$ samtmenge zulassen, ohne daß es jemals zu mikroskopisch inhomogenen Ausscheidungen käme.

13 E.Schulz u. G. W as sermann, Z. Metallkunde 32, 415 [1940], finden beim Zerfall übersättigter Aluminium-Mischkristalle mit Ausnahme eines möglichen Sonderfalls auch nur mikroskopisch homogene Ausscheidungen. 